\title{
Dust tracking techniques applied to the STARDUST facility: First results
}

\author{
A. Malizia ， M. Camplani , M. Gelfusa , I. Lupelli ，M. Richetta , L. Antonelli \\ F. Conetta , D. Scarpellini ，M. Carestia , E. Peluso ,C. Bellecci , L. Salgado ，P. Gaudio
}

\section{H I G H L I G H T S}

- Use of an experimental facility, STARDUST, to analyze the dust resuspension problem inside the tokamak in case of loss of vacuum accident.

- PIV technique implementation to track the dust during a LOVA reproduction inside STARDUST.

- Data imaging techniques to analyze dust velocity field: first results and data discussion.

\begin{abstract}
A B S T R A C T
An important issue related to future nuclear fusion reactors fueled with deuterium and tritium is the creation of large amounts of dust due to several mechanisms (disruptions, ELMs and VDEs). The dust size expected in nuclear fusion experiments (such as ITER) is in the order of microns (between 0.1 and $1000 \mu \mathrm{m}$ ). Almost the total amount of this dust remains in the vacuum vessel (VV). This radiological dust can re-suspend in case of LOVA (loss of vacuum accident) and these phenomena can cause explosions and serious damages to the health of the operators and to the integrity of the device. The authors have developed a facility, STARDUST, in order to reproduce the thermo fluid-dynamic conditions comparable to those expected inside the VV of the next generation of experiments such as ITER in case of LOVA. The dust used inside the STARDUST facility presents particle sizes and physical characteristics comparable with those that created inside the VV of nuclear fusion experiments. In this facility an experimental campaign has been conducted with the purpose of tracking the dust re-suspended at low pressurization rates (comparable to those expected in case of LOVA in ITER and suggested by the General Safety and Security Report ITER-GSSR) using a fast camera with a frame rate from 1000 to 10,000 images per second. The velocity fields of the mobilized dust are derived from the imaging of a two-dimensional slice of the flow illuminated by optically adapted laser beam. The aim of this work is to demonstrate the possibility of dust tracking by means of image processing with the objective of determining the velocity field values of dust re-suspended during a LOVA.
\end{abstract}

\section{Introduction}

By the nature of its operation, an experimental nuclear fusion device generates aerosol particulate and flakes that may affect its safety and operational performance. Matter of this type, usually referred to as dust, is produced in fusion devices by energetic plasma-surface interactions, (disruptions and other events) that cause significant erosion. A sizeable portion of the eroded material does not adhere to surfaces and generates dust, which is capable of being re-suspended in case of events like LOVA. Several mechanisms can be responsible for generation of particulate. Possible mechanisms in magnetic fusion systems include blistering and fracturing of deposited layers, generation of reactive species in edge plasmas, arcing, explosive ejection and brittle destruction of surface imperfections, and nucleation of vaporized materials [1]. If a LOVA occurs in a fusion reactor, buoyancy-driven exchange flows take place at breaches of the vacuum vessel (VV) due to the temperature and pressure difference between the inside and outside of the vacuum vessel. The exchange flows may bring mixtures of activated material and tritium through the breaches. Particle sizes 


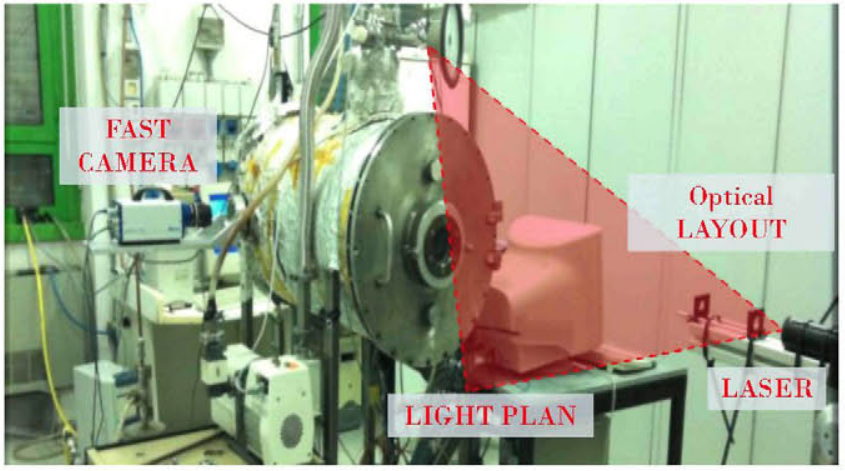

Fig. 1. Experimental apparatus.

are typically small, in the 0.1 micron to $1000 \mu \mathrm{m}$ diameter range. This particle size raises three separate classes of safety concerns [2]. The first concern is that because of the small diameters, these toxic dusts can be easily re-suspended (the decreasing particle diameter leads to dominance of fluid drag $\left(\sim \mathrm{d}^{2}\right)$ over gravity $\left(\sim \mathrm{d}^{3}\right)$ forces, but is important notice that at the smallest of diameters, adhesive forces ( $\sim \mathrm{d})$ dominate and inhibit resuspension).

The second concern is for personnel occupational safety, since these dusts are breathable sizes [21-24]. The third concern is that these dusts are potentially combustible. Several experimental studies [3-19] demonstrated that the exchange flows depend on number, position, length and shape of breaches, pressure and temperature conditions and type of fluid. Therefore, it is very important to investigate the dust re-suspension characteristics during a LOVA event. The Quantum Electronics and Plasma Physics Research Group of University of Rome Tor Vergata (QEP) has developed an experimental facility, STARDUST, that allows the reproduction of thermo fluid-dynamic comparable to those expected in the vacuum vessel (VV) of nuclear fusion plants like ITER [7-14,16-19]. In this work the QEP in collaboration with the Grupo de Tratamiento de Imágenes (GTI) of Universidad Politécnica de Madrid demonstrate the capability of STARDUST to collect images of dust re-suspended during a LOVA and to obtain information about the velocity field of dust re-suspended. The aim of this work is to demonstrate the possibility to use STARDUST not only to analyze the thermo fluid-dynamic behavior in case of LOVA but also the velocity fields characteristics of dust re-suspended. The experimental set-up and first results of the dust tracking techniques applied to this particular case will be shown.

\section{STARDUST-experimental set up}

In the previous experimental campaign QEP demonstrate the capability of STARDUST to replicate thermo fluid-dynamic conditions comparable to those expected inside the VV of nuclear fusion plants such as ITER [7,9,11,13,14,16,18,19] and the dust resuspension fraction in case of LOVA [8-10,12,17]. QEP validated the experimental results by means of CFD codes developed in 2D [7-19]. The new experimental set-up allows a high frequency rate images acquisition of dust re-suspended in case of LOVA inside STARDUST VV. The implemented set-up reproduces a sort of Particle Image Velocimetry (PIV), to obtain instantaneous velocity measurements and related properties of particles within the fluids.

Instead of introducing a tracer in the fluid the authors used the compressed air of STARDUST and illuminate this fluid so that dust particles are visible. The implemented experimental apparatus (Fig. 1) consists of a fast camera [30] and a He-Ne Laser (632.8 nm-30 W) with an optical layout (Fig. 1) to limit the illuminated physical region. The optics consists in a cylindrical lens to convert a light beam into a line and a square lens to convert the line

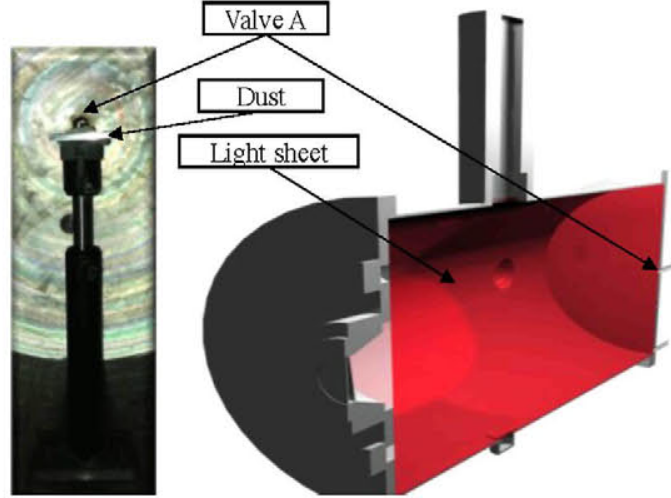

Fig. 2. SS136 placed at the level of Valve A at a distance of $15.5 \mathrm{~cm}$.

into a sheet of light. That sheet illuminates the symmetry plan of the STARDUST VV and the dust placed at the level of Valve A (Fig. 2), used to reproduce a LOVA inside STARDUST at equatorial level $[7,9,11,13]$. The trigger of the fast camera is synchronized $100 \mathrm{~ms}$ before the inlet of air from the flow meter, in order to associate to each frame instantaneous values of: internal pressure, internal temperature, wall temperature and flow rates values automatically collected during the experiment [7-14,16-19]. The dust used previously was SS316 and W [12]. In this experimental campaign the authors used only SS316, with a granulometry from 15 to $45 \mu \mathrm{m}$, see Fig. 3 (Scanning Electron Microscopy characterization has been discussed in [12]) due to its brightness and capability to scatter the light compared to $\mathrm{W}$ that is a dark dust. The motion of the seeding dust has been used to calculate their speed and direction (the velocity field).

\section{Particles detection and tracking}

The proposed experimental setup and the fast camera allow dust particles trajectories detection inside STARDUST during a LOVA reproduction; as it can be noticed from Fig. 4(a), they appear as bright spots on a dark background. The goal of the proposed approach is to detect and track the dust particles in the tank to estimate their velocity and direction during the experiment. The proposed approach presented in Fig. 5 consists of three main steps: Pre-processing (imgProc block) to improve the image quality and ease particle detection and tracking (PartDet and PartTrack blocks). During the pre-processing step histogram equalization $[25,26]$ is applied to increase the images' contrast. This step is fundamental. As Fig. 4(a) shows, the raw data provided by the fast camera do not allow to clearly discriminating moving dust particles with respect to the background. Fig. 4(b) illustrates an example of the contrast-enhanced image; as we can see, moving particles definition is enhanced allowing particle detection and tracking. The spatial resolution is approximately $0.0415 \mathrm{~cm}$ for each pixel.
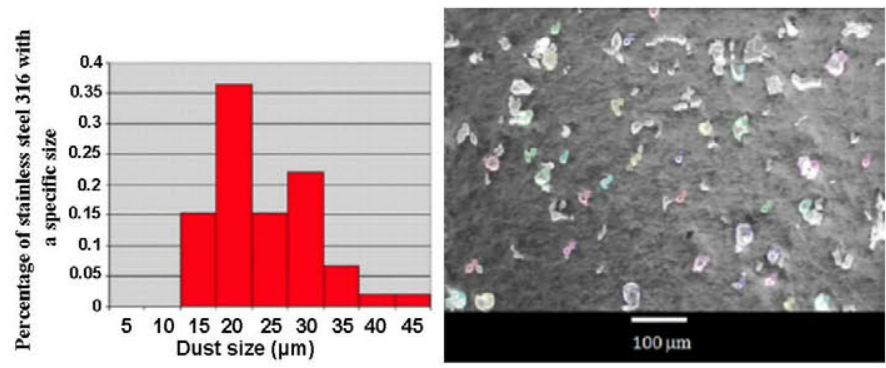

Fig. 3. SEM of stainless steel 316 used inside STARDUST [12]. 


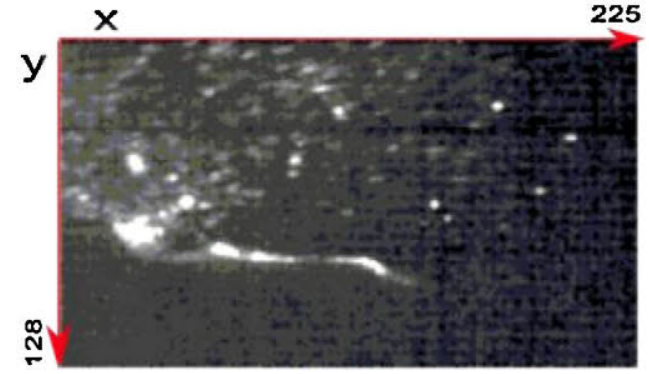

(a)

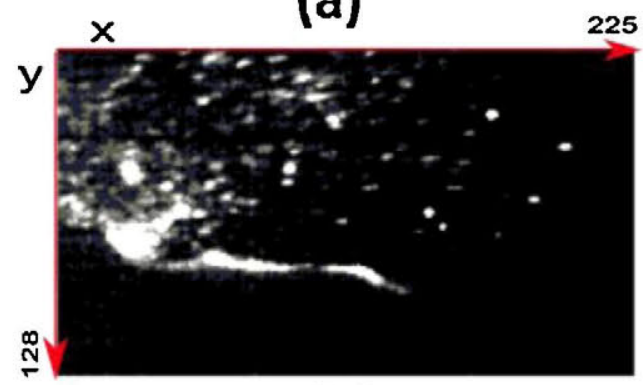

(b)

Fig. 4. Raw image acquired by the fast camera (a), image with enhanced contrast (b), image dimension are in pixels.

The obtained high contrast images (HC_img, Fig. 5) are used to detect the moving particles in the tank, frame by frame (PartDet, Fig. 5): we implement a background model of the scene acquired during the experiment and used it to detect the moving particles. In particular, the background model is based on the Mixture of Gaussian (MoG) background model presented in [27]. The approach is particularly suitable for these applications since it guarantees an accurate per-pixel multimodal background model, capable to reduce the temporal flickering noise of the acquired images (Table 1).

The foreground mask (FG_part in Fig. 5) containing the detected particles with the proposed PartDet, and corresponding to the image in Fig. 3 block, is shown in Fig. 6. A morphological erosion filter (square elements of size 2 is used in our experiments) removes possible false detections; it is worth noting that very small particles (with an area smaller than 4 pixels) can be also discarded.

The obtained foreground connected regions are filtered by considering their area in order to identify the small moving particles. In the large foreground region corresponding to an area densely populated by dust particles, it is not possible to clearly distinguish the several particles that compose it. Once this large region is detected it is tracked separately and its estimated velocity and other parameters are considered representative of all the particles that compose it. These detections are processed by PartTrack step (Fig. 6) to provide an estimation of their trajectory and velocity. In particular, a Kalman Filter based [28] multi-object tracking

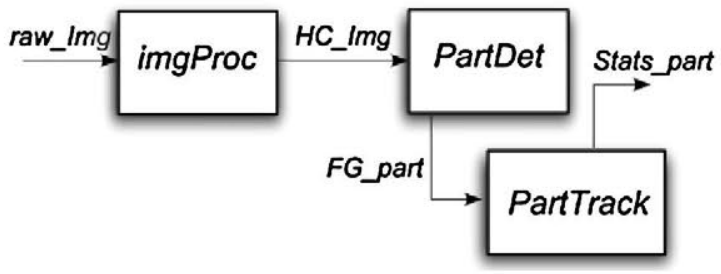

Fig. 5. Block diagram of the particle tracking system.
Table 1

Experimental data collected inside STARDUST.

\begin{tabular}{lllll}
\hline Time $(\mathrm{s})$ & $T_{\text {wall mean }}(\mathrm{K})$ & $T_{\text {env }}(\mathrm{K})$ & $P_{\text {in }}(\mathrm{Pa})$ & FR $(1 / \mathrm{min})$ \\
\hline 0.0744 & 280.13 & 280.46 & 170.92 & 3.18 \\
0.11 & 282.13 & 280.46 & 191.83 & 4.68 \\
\hline
\end{tabular}

1st Column: Time (in second) after the beginning of air flow inlet. 2nd Column: Mean value of wall temperature (in Kelvin) measured by thermocouples. 3rd Column: Mean value of STARDUST internal environment temperature (in Kelvin) measured by thermocouples. 4th Column: Internal pressure inside STARDUST (in Pascal). 5th Column: Flow rate of pressurized air flowed inside STARDUST ( $1 / \mathrm{min})$.

strategy is applied. The Kalman filter is a recursive data processing algorithm that generates optimal estimates of the system state for linear systems with white Gaussian errors.

In our case the state of the system (each particle) is composed by three variables: position, velocity and acceleration. The Kalman Filter predicts for each particle its state in the successive frames. These predicted positions and the detected particles positions are then associated by considering the geometrical distance of the particles centroids in the image plane. The Hungarian algorithm [29] is used to solve the assignment problem, allowing tracking the same particles along the frames. The advantages of using the Kalman filter are twofold since it allows to recover miss-detected particles (thanks to the prediction phase) and to accurately model their individual dynamic behavior.

In particular, according to the experimental conditions, we use a state model based on a constant acceleration of the particles during the first seconds of vacuum rupture. It is worth noting that the Kalman-filter based tracking does not depend on the particles' size. The proposed approach initializing a new Kalman tracker for each detected particle that has not been associated with any of the already tracked particles. On the contrary, tracked particles that have not been associated with new detection for a long time are deleted from the multi-object tracking framework. An example of a tracked particle along different frames is shown in Fig. 7. The first frame, in which the particle is detected (see the red squared mark in the Image) is shown in Fig. 7(a) and the last frame in which the particle appears in Fig. 7(b) (marked in red). It has to be noticed that the particle trajectory reported in Fig. 7 is obtained by joining the particles position identified in the intermediate frames (25 in this example). The dust from frame (a) to frame (b) in Fig. 7 seems to be partially disappeared, this is because an amount of dust detected in frame (a) has been re-suspended out of the sheet of light created with the laser source. It is not possible collect the scattering of this amount with the fast camera. The algorithms developed allow to track particle of cluster of dust that re-suspend inside the sheet of light and are able to scatter light on the fast camera's CCD.

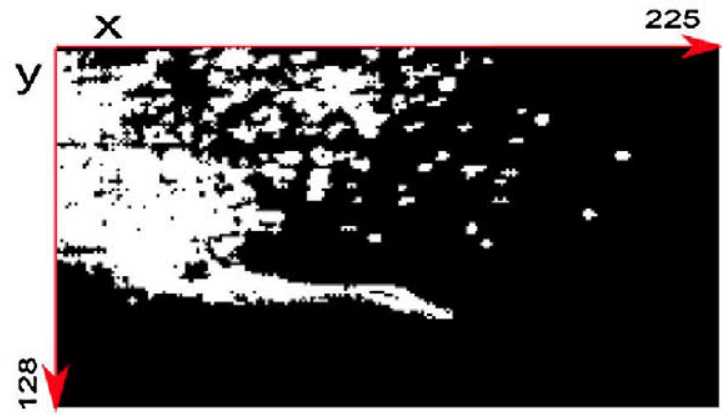

Fig. 6. Detected dust particles. A portion of image is shown. The dimensions are in pixels. 


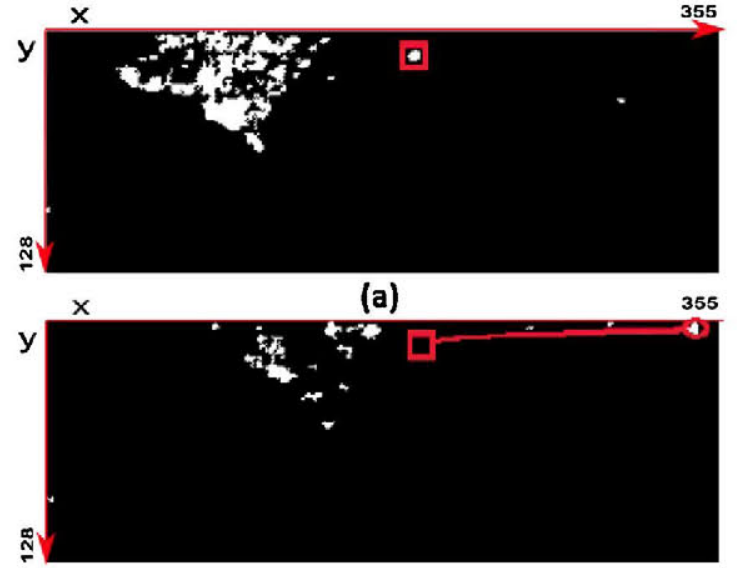

(b)

Fig. 7. Example of a tracked dust particle. A portion of image is shown. The dimen sions are in pixels. (For interpretation of the references to color in this figure legend, the reader is referred to the web version of the article.)

\section{Data discussion}

The sequence analyzed is the one at $5000 \mathrm{fps}$ simulating a LOVA at $300 \mathrm{~Pa} / \mathrm{s}(27 \mathrm{l} / \mathrm{min}$ of flow rate see [8-14,16-20] for more detail about the experiment). It is worth noting that each pixel corresponds, for this acquisition, to approximately $0.0415 \mathrm{~cm}$. The dust is tracked between the frames 0 and 600 that means in a time range (from the beginning of the air inlet inside STARDUST) $0-0.12 \mathrm{~s}$. After $0.11 \mathrm{~s}$ all the dust placed on the tray is completely mobilized. In Fig. 8 (in a time range from $0.074 \mathrm{~s}$ to $0.11 \mathrm{~s}$ ) dust particle moves at a maximum velocity of approximately $203 \mathrm{~m} / \mathrm{s}$.

By data images techniques the velocity values of dust particles mobilized are calculated considering a time step of $0.002 \mathrm{~s}$ ( 10 images). The results of dust velocity have been compared with the velocity values of the air measured, by the mean of a pressure transducer [13], in the same position of dust experimental conditions and temporal interval. The comparison is shown in Fig. 8. The time range of the images sequence analyzed correspond to the following experimental data collected:

It is evident that the values of velocity decrease after frames 522 (the dust is completely mobilized). That means that almost all the mobilization of dust happens $1-1.8 \mathrm{~s}$ before that of the maximum pressurization rate $(300 \mathrm{~Pa} / \mathrm{s}$ at $271 / \mathrm{min})$ and the connected maximum air velocity values is reached.

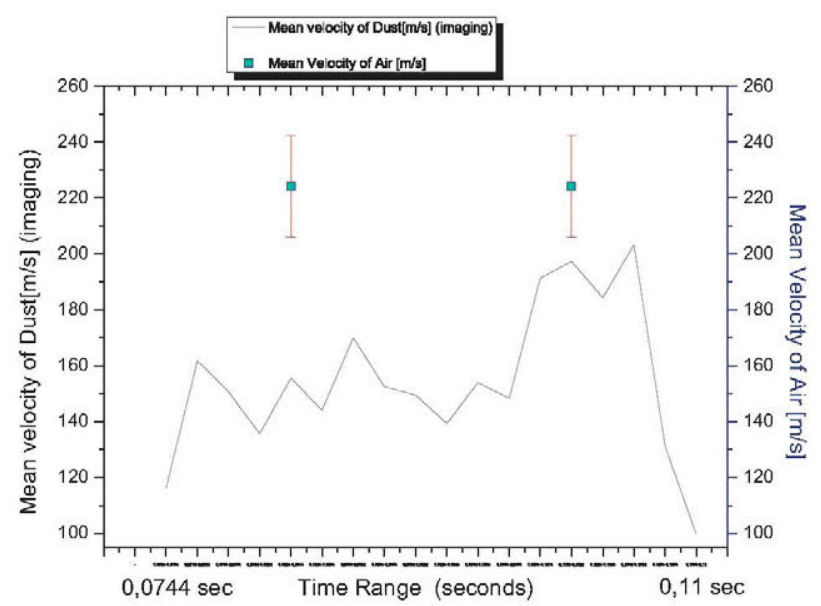

Fig. 8. Dust velocity values measured at the beginning of air flow inlet compared to the velocity values of air.

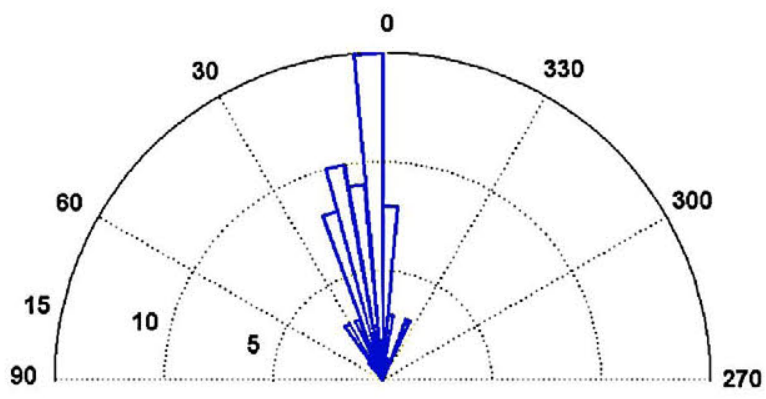

Fig. 9. Dust principal directions during the mobilization.

The authors applied the techniques also to obtain the principal directions of the dust respect the horizontal plane of the tray (Fig. 9). The principal trajectories lie between 15 and 30 degree, and they are evaluated with respect to the image plane coordinate system.

\section{Conclusions and future developments}

The authors developed an experimental set-up to reproduce a LOVA that could be expected in a nuclear fusion plant like ITER. They acquired, with a high speed camera and a proper optical apparatus, image sequences of dust mobilized during the LOVA reproduction. The authors also developed a computer vision approach to derive data from the images by means of an image processing technique. In particular: dust velocity (absolute and relative); dust direction. We face the problem of a complete mobilization of dust before the maximum pressurization rate is reached. In the first phase of experiment dust velocities values are lower than the ones of pressurized air measured in the same point and at the same time with pressure transducer and it has to be investigated. In order to associate the results from data imaging to the thermo fluid-dynamics property of the inflow air during the maximum velocity regime these future upgrades have to be implemented: (a) increase the field of view inside STARDUST by introducing new windows. (b) Use a continuum laser that emits in the green or blue region to better exploit the camera quantum efficiency. (c) Flow the air from the divertor region and collect images in order to reduce the direct impact of air on the dust so its velocity. (d) Compare the velocities with those recovered with lower pressurization rates. (e) Analyze the effect of adhesive forces that for the small particle dominate and inhibit the resuspension. (f) Develop a scaling analysis to better reproduce a LOVA conditions inside the experimental facility.

\section{References}

[1] J.P. Sharpe, D.A. Petti, H.-W. Bartels, A review of dust in fusion devices: impli cations for safety and operational performance, Fusion Eng. Des. 63-64 (2002) 153-163.

[2] K. Takase, T. Kunugi, M. Shibata, Y. Seki, Temperature distributions in a Tokamak vacuum vessel of fusion reactor after the loss-of-vacuum events occurred, Fusion Eng. Des. 42 (1998) 83-88.

[3] W.G. Brown, Natural convection through rectangular openings in partition-2 horizontal partition, Int. J. Heat Mass Tran. 5 (1962) 859-871.

[4] K. Takase, T. Kunugi, Y. Seki, Effects of breach area and length to exchange flow rates under the LOVA conditions in a fusion reactor, Fusion Technol. 30 (1996) $1459-1464$.

[5] K. Takase, T. Kunugi, M. Ogawa, Y.Seki, Experimental study on buoyancy-driven exchange flows through breaches of a Tokamak vacuum vessel in a fusion reactor under the loss-of vacuum event condition, Nucl. Sci. Eng. 125 (1997) $223-231$.

[6] K. Takase, Three-dimensional numerical simulations of dust mobilization and air ingress characteristics in a fusion reactor during a LOVA event, Fusion Eng. Des. 54 (2001) 605-615.

[7] P. Gaudio, A. Malizia, I. Lupelli, Experimental and numerical analysis of dust resuspension for supporting chemical and radiological risk assessment in a nuclear fusion device, in: International Conference on Mathematical Models for Engineering Science - Proceedings, 2010, pp. 134-147. 
[8] P. Gaudio, A. Malizia, I. Lupelli, RNG $k-e$ modelling and mobilization experiments of loss of vacuum in small tanks for nuclear fusion safety applications, Int. J. Syst. Appl. Eng. Dev. 5 (2011) 287-305, ISSN: 2074-1308.

[9] M. Benedetti, P. Gaudio, I, Lupelli, A. Malizia, M.T. Porfiri, M. Richetta, Large eddy simulation of loss of vacuum accident in STARDUST facility, Fusion Eng. Des. $88(9-10)(2013)$ 2665-2668.

[10] M. Benedetti, P. Gaudio, I, Lupelli, A. Malizia, M.T, Porfiri, M. Richetta, Influence of temperature fluctuations. Measured by numerical simulations, on dust resuspension due to L. O. V.As, Int. J. Syst. Appl. Eng. Dev. 5 (2011) 718-727, ISSN: 2074-1308.

[11] C. Bellecci, P. Gaudio, I. Lupelli, A. Malizia, M.T. Porfiri, R. Quaranta, et al., Validation of a loss of vacuum accident (LOVA) computational fluid dynamics (CFD) model, Fusion Eng. Des. 86 (9-11) (2011) 2774-2778.

[12] C Bellecci, P. Gaudio, I. Lupelli, A Malizia, M.T. Porfiri, R. Ouaranta, et al. STARDUST experimental campaign and numerical simulations: influence of obstacles and temperature on dust resuspension in a vacuum vessel under LOVA, Nucl. Fusion 51 (5) (2011) (053017).

[13] C. Bellecci, P. Gaudio, I. Lupelli, A. Malizia, M.T. Porfiri, R. Quaranta, et al., Loss of vacuum accident (LOVA): comparison of computational fluid dynamics (CFD) flow velocities against experimental data for the model validation, Fusion Eng. Des. 86 (4-5) (2011) 330-340.

[14] M. Benedetti, P. Gaudio, I. Lupelli, A. Malizia, M.T. Porfiri, M. Richetta, Scaled experiment for loss of vacuum accidents in nuclear fusion devices: experimental methodology for fluid-dynamics analysis in STARDUST facility, in: Recent Researches in Mechanics - Proc. of the 2nd Int. Conf. on FLUIDSHEAT11, TAM'11, Proc. of the 4th WSEAS Int. Conf. UPT'11, CUHT'11, 2011, pp. 142-147.

[15] T. Pinna, L.C. Cadwallader, G. Cambi, S. Ciattaglia, S. Knipe, F. Leuterer, et al., Operating experiences from existing fusion facilities in view of ITER safety and reliability, Fusion Eng. Des. 85 (7-9) (2010) 1410-1415.

[16] C. Bellecci, P. Gaudio, I. Lupelli, A. Malizia, M.T. Porfiri, R. Quaranta, et al., Experimental mapping of velocity flow field in case of L.O.V.A inside stardust facility, in: 37th EPS Conference on Plasma Physics 2010, vol. 2, 2010, pp. 703-706.

[17] P. Gaudio, A. Malizia, I. Lupelli, Experimental and numerical analysis of dust resuspension for supporting chemical and radiological risk assessment in a nuclear fusion device, in: Conference Proceedings - International Conference on Mathematical Models for Engineering Science (MMES' 10), Puerto De La Cruz, Tenerife, 30/11/2010 - 30/12/2010, 2010, pp. 134-147, ISBN/ISSN: 978 $960-474-252-3$.
[18] C. Bellecci, P. Gaudio, I. Lupelli, A. Malizia, M.T. Porfiri, R. Quaranta, et al., Characterization of divertor influence in case of LOVA: CFD analysis of stardust experimental facility, 36th EPS Conference on Plasma Physics 2009, EPS 2009 - Europhysics Conference Abstracts 33E1 (2009) 266-269.

[19] C. Bellecci, P. Gaudio, I. Lupelli, A. Malizia, M.T. Porfiri, M. Richetta, Dust mobilization and transport measures in the STARDUST facility, Hersonissos, Crete, Greece, 9-13, vol. ECA June 2008, EPS2008 Proceedings, 35th EPS Conference on Plasma Physics 32 (2008) (P-1.175)

[20] R. Gallo, P. De Angelis, A. Malizia, F. Conetta, D. Di Giovanni, L. Antonelli, et al. Development of a georeferencing software for radiological diffusion in order to improve the safety and security of first responders, Def. S\&Tech. Bull. 6 (1) (2013) 21-32.

[21] A. Malizia, R. Quaranta, R. Mugavero, R. Carcano, G. Franceschi, Proposal of the prototype RoSyD-CBRN, a robotic system for remote detection of CBRN agents, Def. S\&T Tech. Bull. 4(1)(2011) 64-76.

[22] A. Malizia, I. Lupelli, F. D’Amico, A. Sassolini, A. Fiduccia, A.M. Quarta, et al. Comparison of software for rescue operation planning during an accident in a nuclear power plant, Def. S\&T Tech. Bull. 5 (1)(2012) 36-45.

[23] A. Malizia, R. Quaranta, R. Mugavero, R. Carcano, G. Franceschi, Proposal of the prototype RoSyD-CBRN, a robotic system for remote detection of CBRN agents, Def. S\&T Tech. Bull. 4 (2011) 64-76, ISSN: 1985-6571.

[24] O. Cenciarelli, A. Malizia, M. Marinelli, S. Pietropaoli, R. Gallo, F. D’Amico, et al. Evaluation of biohazard management of the Italian national fire brigade, Def. S\&T Tech. Bull. 6 (1) (2013) 33-41.

[25] A. Murari, M. Camplani, B, Cannas, D. Mazon, F. Delaunay, P. Usai, et al., Algo rithms for the automatic identification of MARFE and UFO in JET database of visible camera videos, IEEE Trans. Plasma Sci. 38 (2) (2010) 3409-3418.

[26] R.C. Gonzalez, R.E. Woods, Digital Image Processing, Addison Wesley, USA, Gatesmark Publishing, 1992.

[27] C. Stauffer, W.E.L. Grimson, Adaptive background mixture models for real-time tracking, in: IEEE Conference on Computer Vision and Pattern Recognition, vol. 24, 1999, pp. 6-252.

[28] A. Yilmaz, O. Javed, M. Shah, Object tracking, ACM Comput. Surv. 38 (4) (2006)

[29] J. Munkres, Algorithms for the assignment and transportation problems, J. Soc Ind. Appl. Math. 5 (1) (1957) 32-38.

[30] CAMRECORD 1000, Optronis, datasheet (http://optronis.dev.tandemkommunikation.de/fileadmin/user_upload/download/CamRecord1000_engl. pdf). 